\title{
Enhanced Bidirectional Connectivity of the Subthalamo-pallidal Pathway in 6-OHDA-mouse Model of Parkinson's Disease Revealed by Probabilistic Tractography of Diffusion-weighted MRI at 9.4T
}

\author{
A-Yoon $\mathrm{Kim}^{1}$, Chiwoo $\mathrm{Oh}^{2}$, Hyung-Jun $\mathrm{Im}^{2}$ and Hyeon-Man Baek ${ }^{1,3 *}$ \\ ${ }^{1}$ Department of Health Science and Technology, GAIHST, Gachon University, Incheon 21936, ${ }^{2}$ Department of \\ Transdisciplinary Studies, Graduate School of Convergence Science and Technology, Seoul National University, Seoul 16229, \\ ${ }^{3}$ Lee Gil Ya Cancer \& Diabetes Institute, Gachon University, Incheon 21999, Korea
}

\begin{abstract}
An important challenge in Parkinson's disease (PD) based neuroscience and neuroimaging is mapping the neuronal connectivity of the basal ganglia to understand how the disease affects brain circuitry. However, a majority of diffusion tractography studies have shown difficulties in revealing connections between distant anatomic brain regions and visualizing basal ganglia connectome. In this current study, we investigated the differences in basal ganglia connectivity between 6-OHDA induced ex-vivo PD mouse model and normal ex-vivo mouse model by using diffusion tensor imaging tractography from diffusion-weighted images obtained with a high resolution 9.4 T MR scanner. Connectivity pattern of the basal ganglia were compared between five 6-OHDA and five control ex-vivo mouse brains using results of probabilistic tractography generated with PROBTRACKX. When compared with control mouse, 6-OHDA mouse showed significant enhancements to motor territory-related subthalamopallidal and pallido-subthalamic connectivity. Multi-fiber tractography combined with diffusion MRI data has the potential to help recognize the abnormalities found in connectivity of psychiatric and neurologic disease models.
\end{abstract}

Key words: Basal ganglia, Parkinson's disease, Diffusion MRI, Probabilistic tractography, Globus pallidus, Subthalamic nucleus

\section{INTRODUCTION}

Parkinson's disease (PD) is a common neurodegenerative disease of the central nervous system that primarily affects the motor system [1]. The most distinct motor symptoms of PD are gait impairments, tremor, muscular rigidity, slowness of movement as well as non-motor dysfunctions such as behavioral and cognitive impairments [2]. These symptoms of PD are caused by progres-

Received December 27,2019, Revised February 15,2020,

Accepted February 15, 2020

* To whom correspondence should be addressed. TEL: 82-32-899-6678, FAX: 82-32-899-6677 e-mail:hmbaek98@gachon.ac.kr sive loss of dopamine-producing cells in the substantia nigra pars compacta $(\mathrm{SNc})$, one of five nuclei in the basal ganglia that plays a key role in voluntary movement $[2,3]$. As a result, dopamine levels are lowered, inhibiting innervation of basal ganglia output and dysfunction within the cortico-basal ganglia thalamic pathway [4]. The basal ganglia is a network of subcortical nuclei, consisting of the striatum, internal and external globus pallidus (GPi/GPe), subthalamic nucleus (STN), substantia nigra compacta $(\mathrm{SNc})$ and substantia nigra reticulata $(\mathrm{SNr})$ [5]. Neural projections of the striatum involved in the motor system are segregated into direct and indirect pathways. It is believed that dopamine secreted from SNc activates striatal D1 neurons in the direct pathway and inhibits the striatal D2 neurons in the indirect pathway. In PD, dopamine depletion leads to diminished inhibitory direct pathway output 
and increased excitatory indirect pathway output onto GPi/SNr [6]. However, such connections influencing the motor system are not completely analyzed in normal and disease state mouse models.

Diffusion tensor imaging (DTI) measures the diffusion of water molecule protons which are then used to analyze the changes in microstructure within brain regions of both animals and humans [7]. Developments in diffusion MRI (dMRI) and tractography techniques have offered the possibility of non-invasively mapping neural connections in animal and human brain by reconstructing fiber pathway through each voxel containing directional diffusion information $[8,9]$. Table 1 shows diffusion MR studies that have been used to investigate connectivity between brain regions, including basal ganglia, and identify neurodegeneration in myelin or other indicators $[8,10-16]$. Using dMRI-based tractography, several studies have generated connectivity matrices representing connectivity strength by counting the number of fibers connecting each brain region $[10,17]$. Therefore, diffusion MRI analysis is considered to be a very useful technique in identifying brain connectome through the calculation of structural connectivity.

Despite such benefits, previous diffusion tractography studies have shown difficulties in revealing connections between brain regions in humans (Table 1). A study regarding human brain connectivity showed connectivity between the cerebellum and the GPi as well as the SN; however, the study showed that it was dif-

Table 1. List of previously reported studies associated with diffusion MR imaging

\begin{tabular}{|c|c|c|c|c|c|}
\hline Authors & Secies & Year & Scanner & Data/Analysis program & Finding \\
\hline Calabrese et al. [10] & $\begin{array}{l}\text { 2-Wild type }(\mathrm{C} 57 \mathrm{BL} / 6) \\
\text { Mice }\end{array}$ & 2015 & $9.4 \mathrm{~T}$ & $\begin{array}{l}\text { Diffusion MR image } \\
\text { Program: ANTX/FSL }\end{array}$ & $\begin{array}{l}\text { Ipsilateral connectivity was higher than } \\
\text { contralateral connectivity. Significant } \\
\text { correlation was detected between ABA } \\
\text { neuronal tracer data and diffusion } \\
\text { tractography data. }\end{array}$ \\
\hline Cong et al. [11] & $\begin{array}{l}6 \text { MitoPark Mice (Par- } \\
\text { kinson disease)/ } 9 \text { wild- } \\
\text { type }(\mathrm{C} 57 \mathrm{BL} / 6) \text { Mice }\end{array}$ & 2016 & $7 \mathrm{~T}$ & $\begin{array}{l}\text { Diffusion MR image/ T2* map/ } \\
\text { Cerebral blood flow MR images } \\
\text { Program: Matlab }\end{array}$ & $\begin{array}{l}\text { T2* of the MitoPark group showed sig- } \\
\text { nificant reductions in the striatum and } \\
\text { substantia nigra compared with control } \\
\text { groups. ADC values of the MitoPark } \\
\text { group showed significant reductions } \\
\text { in the SN compared with the control } \\
\text { group. }\end{array}$ \\
\hline Hübner et al. [12] & $\begin{array}{l}\text { Cuprizone treated } \\
\text { (CUPR) C57BL/6N } \\
\text { mice }\end{array}$ & 2017 & $7 \mathrm{~T}$ & $\begin{array}{l}\text { rsfMRI data/ T2-weighted im- } \\
\text { age/ Diffusion MR images }\end{array}$ & $\begin{array}{l}\text { CUPR induced pathology decreases } \\
\text { average functional connectivity strength } \\
\text { within the whole functional connectiv- } \\
\text { ity matrix. }\end{array}$ \\
\hline Perlbarg et al. [13] & $\begin{array}{l}15 \text { Sprague Dawley rats } \\
\text { (10 rats: } 6 \text {-OHDA injec- } \\
\text { tion. } 5 \text { rats: physiologi- } \\
\text { cal solution injection) }\end{array}$ & 2018 & $11.7 \mathrm{~T}$ & $\begin{array}{l}\text { T2-weighted image/Multi-slice } \\
\text { echo-planar image/ Diffusion } \\
\text { MR image } \\
\text { Program: MRtrix3 package }\end{array}$ & $\begin{array}{l}\text { There was increased FA value in the } \\
\text { ipsilateral and contralateral striatum. }\end{array}$ \\
\hline Lenglet et al. [14] & 4 healthy humans & 2012 & $7 \mathrm{~T}$ & $\begin{array}{l}\text { T1-weighted images/T2- } \\
\text { weighted images/Susceptibility- } \\
\text { weighted images/Diffusion MR } \\
\text { image } \\
\text { Program: FSL }\end{array}$ & $\begin{array}{l}\text { Connections of STN to GP were higher } \\
\text { for GPi than for GPe. STN-striatum } \\
\text { connections were weak. }\end{array}$ \\
\hline Milardi et al. [15] & 15 human subjects & 2016 & $3 \mathrm{~T}$ & $\begin{array}{l}\text { 3DT1-weighted image/3DT2- } \\
\text { weighted image/Diffusion MR } \\
\text { image } \\
\text { Program: FLIRT of FSL }\end{array}$ & $\begin{array}{l}\text { This study found the presence of direct } \\
\text { connections between } \mathrm{SN} \text { and Dentate } \\
\text { nucleus. Dentate was widely projected } \\
\text { to the GP.. }\end{array}$ \\
\hline Plantinga et al. [16] & $\begin{array}{l}\text { A formalin fixed human } \\
\text { brain specimen }\end{array}$ & 2016 & $7 \mathrm{~T}$ & $\begin{array}{l}\text { Diffusion MR image } \\
\text { Program: FSL/ITK-SNAP soft- } \\
\text { ware/MRtrix }\end{array}$ & $\begin{array}{l}\text { Direct connections within the basal gan- } \\
\text { glia (STN, STN-GP, STN-SN, SNr-SNc } \\
\text { etc.) were shown in this study. STN-GPi } \\
\text { connections were fewer than the STN- } \\
\text { SNr connections, that reflect a relatively } \\
\text { important role for the STN-SNr connec- } \\
\text { tion in human. }\end{array}$ \\
\hline Pujol et al. [8] & 5 healthy subjects & 2017 & $3 \mathrm{~T}$ & $\begin{array}{l}\text { T1-weighted image/T2-weighted } \\
\text { image/ Diffusion MR image } \\
\text { Program: FSL/MRtrix }\end{array}$ & $\begin{array}{l}\text { This study investigated the white matter } \\
\text { connectivity between the STN and GP. } \\
\text { A number of fibers interconnecting GPi } \\
\text { arose from the ventral-medial STN. }\end{array}$ \\
\hline
\end{tabular}


ficult to distinguish between the $\mathrm{SNc}$ and $\mathrm{SNr}$ on the basis of MRI signal [15]. In another study, certain pathways (such as the STNthalamus) could not be visualized because of the proximity of the brain regions [14]. Although experimental neurologic connectivity methods still represent a gold-standard for neuroanatomical tracttracing studies of basal ganglia, such methods generally have limitations with low signal-to-noise ratio (SNR) and low resolution of diffusion MRI, resulting in difficulties with accurately fitting complex fibers in voxels [18]. Furthermore, there are many challenges with human in vivo MRI data acquisition including susceptibility artifacts and motion $[19,20]$.

We used three distinct technologies to improve these limitations: 1) high-field animal MR scanners such as $9.4 \mathrm{~T}$ are equipped with strong imaging gradients, allowing strong diffusion gradient pulse and high spatial resolution [21]; 2) imaging of ex-vivo mouse brain can be treated with MR contrast agents. The present view of neuroscience is largely based on MRI data from rodents and non-human primates using various tracing methods. Many mouse DWI studies have obtained higher quality images with less motion artifacts by using ex-vivo mouse brain treated with contrast agents [22, 23]; 3) diffusion MR-based probabilistic tractography can offer increased susceptibility for detecting indistinctive fiber pathways in disease and/or normal state models [24]. Using probabilistic tractography to calculate structural connectivity over deterministic tractography can help account for uncertainty or distributed connectivity [25].

Mouse PD acute toxin models such as 6-hydroxydopamine (6OHDA) and 1-methyl-4-phenyl-1,2,3,6-tertrahydropyridine (MPTP) can mimic features of PD humans [26]. The 6-OHDA mouse model has proven to be especially valuable for understanding the mechanisms underlying PD symptoms due to the model being able to emulate the changes in basal ganglia circuit and pharmacology observed in PD patients [27].

The neuronal connectivity of the mouse brain has been explored more thoroughly than any other mammalian disease model. The Allen Brain Atlas (ABA), which contains the mesoscale neuronal tracer connectome of the mouse brain, is one of the most quantitative and comprehensive reference for mouse brain connectome [10]. This connectivity atlas includes a database of nearly 500 anterograde viral neuronal tracer projections in wild-type C57BL/6 mouse brains representing axonal injection projections $[10,28]$. Therefore, the ABA neural tracer maps can be used as important information in examining the similarities as well as differences between diffusion-based tractography and ABA-based neuronal tracer data in the mouse brain.

Here, our aim was to investigate the tractography of basal ganglia (caudoputamen (CP), STN, SNc, SNr, GPe, GPi) and thalamus using dMRI of 5 ex-vivo PD mouse brains and 5 control mouse brains. The current study provides new information regarding 1) segmentation and visualization of basal ganglia pathway, 2) reconstruction of fiber tractography pathways connecting 6 regions, 3 ) quantification of the probabilistic tractography connectivity of each pathway, 4) comparison of diffusion tractography with ABA neuronal tracer data in basal ganglia regions of mouse brain. Data presented in this study can serve as a reference database for future brain connectivity studies in the mouse brain.

\section{MATERIALS AND METHODS}

\section{Animal preparation}

All animal experiments and procedures were performed in compliance with the Lee Gil Ya Cancer and Diabetes Institutional Center of Animal Care and Use. Ten male C57BL/6N mice (The Jackson Laboratory, Bar Harbor, ME, USA, 8 weeks old) were chosen for this study. Under surgical anesthesia (2 3\%, Forane; JW Pharmaceutical, Republic of Korea), five C57BL/6N mouse ( 8 weeks old) were anesthetized by intramuscular injection of $10 \mu \mathrm{L}$ of $5 \%$ zoletil 50 (Virbac, Carros, France) and 2\% Rompun (Bayer, Leverkusen, Germany) solution (2:1). The head of mouse was fixed using ear bars in a stereotaxic frame apparatus (David Kopf, CA, USA). The scalp was sterilized using povidone solution. Bregma was exposed through vertical incision and 6-OHDA (Sigma of $3 \mathrm{mg} / \mathrm{mL}$ saline with $0.2 \%$ ascorbic acid) was injected into the right striatum in five $\mathrm{C} 57 \mathrm{BL} / 6 \mathrm{~N}$ mouse [anteroposterior (AP) $0.4 \mathrm{~mm}$, mediolateral (ML) $1.8 \mathrm{~mm}$, dorsoventral (DV) $3.5 \mathrm{~mm}$ from bregma] at a rate of $0.5 \mu \mathrm{L} / \mathrm{min}$ (total volume $2.5 \mu \mathrm{L}$ ) using a Hamilton syringe (26 G). The scalp was sutured using 6-0 surgical suture. The control group was injected with an equal volume of phosphate-buffered saline (PBS) at the same coordinates.

3 weeks after injection, ten mice were perfused with 10\% sucrose solution and fixed with $4 \%$ paraformaldehyde (PFA, SigmaAldrich, St Louis, MO, USA) and 0.1\% Magnevist ${ }^{\circledR}$ (Bondrone, Dongkook, Republic of Korea) in Phosphate-buffered saline (PBS, LB004-01, Welgene Inc., Republic of Korea). After perfusion, mice were decapitated and had skin/muscle removed from the skull. The brains with the intact skull were stored in 4\% PFA and $0.1 \%$ Magnevist ${ }^{\circledR}$ in PBS at $4^{\circ} \mathrm{C}$ for 16 hours. Then, the brain samples were incubated in $0.1 \%$ Magnevist ${ }^{\circledR}$ in PBS for 24 hours, the solution was replaced and the samples were incubated for 3 days. Prior to ex-vivo scanning, all brain samples were placed in custom-made tube for MRI compatibility and soaked in fomblin (Y25/6, Solvay, Brussels, Belgium). 


\section{Data acquisition}

The extracted brain samples were scanned with a 9.4T animal MR scanner (Bruker Biospin, Ettlingen, Germany) and a four-channel receive-only phased array head coil for receiving. The T2-weighted MR images were obtained using a 3D Turbo RARE T2 sequence (repetition time $(\mathrm{TR})=1,800 \mathrm{~ms}$; echo time $(\mathrm{TE})=33.6 \mathrm{~ms}$; flip angle $=90^{\circ}$; field of view $(\mathrm{FOV})=1.2 \times 1.2 \times 1.56$ $\mathrm{cm}^{3}$; matrix $=240 \times 240 \times 156$; bandwidth $=1,400 \mathrm{~Hz}$, total imaging scan time $=1$ hour $43 \mathrm{~min}$ ) and the diffusion tensor images were obtained using 2D EPI diffusion tensor sequence $(\mathrm{TR}=3,000 \mathrm{~ms}$; $\mathrm{TE}=30 \mathrm{~ms}$; flip angle $=90^{\circ} ; \mathrm{FOV}=1.8 \times 1.8 \mathrm{~cm}$; matrix $=90 \times 90$; band width $=1,400 \mathrm{~Hz}$, slice thickness $=0.2 \mathrm{~mm}$; b-value $=3,003 \mathrm{~s} / \mathrm{mm}^{2}$; diffusion directions $=30$; total imaging scan time $=2 \mathrm{hr} 6 \mathrm{~min}$ ).

\section{Anatomic segmentation}

The basal ganglia structures, including CP, GPe, GPi, SNc, SNr and STN were segmented using Atlas Normalization Toolbox using elastiX (ANTX) [29], a MATLAB (MathWorks, Natick, MA, USA) toolbox and FSL (Version 6.0; www.fmrib.ox.ac.uk/fsl) [10]. The obtained T2 and DTI data were converted from DICOM to Neuroimaging Informatics Technology Initiative (NIFTI) format with ANTX. b0 images from the converted data were extracted for structural segmentation using fslroi command of FSL [30]. Next, Allen mouse brain mask labels with left and right hemisphere distinctions were generated using mask-generator in MATLAB toolbox ANTX.

\section{Data processing}

Data processing steps are represented in Fig. 1. A binary mouse brain mask was generated from the b0 image by linearly aligning Allen mouse whole brain masks from Allen template space to native DTI space with FMRIB's Linear Image Registration Tool (FLIRT) $[31,32]$. Registrations were visually inspected with FSL's fsleyes. After registration, all basal ganglia masks were registered to the Allen brain atlas template using FSL's FLIRT transformation. Next, DWI denoising step to improve SNR of data was performed to reduce the influence of thermal noise by applying redundancy in the diffusion data in MRtrix [33]. The denoised DTI images were corrected for eddy currents using FSL's eddycorrect, a tool for correcting distortion and motion [34]. After eddy correction, DTI images were corrected for B1 field inhomogeneities by using dwibiascorrect in MRtrix [35]. Fitting of diffusion tensor on corrected DTI data was done using dtifit of FMRIB's diffusion toolbox. FA and ADC values of DTI data were calculated using FSL's fslmeants, represented in Supplementary data. The volume of each 10 bilateral registered brain masks was calculated with the volume function (-V) using FSL's fslstats [36]. Bayesian Estimation of Diffusion

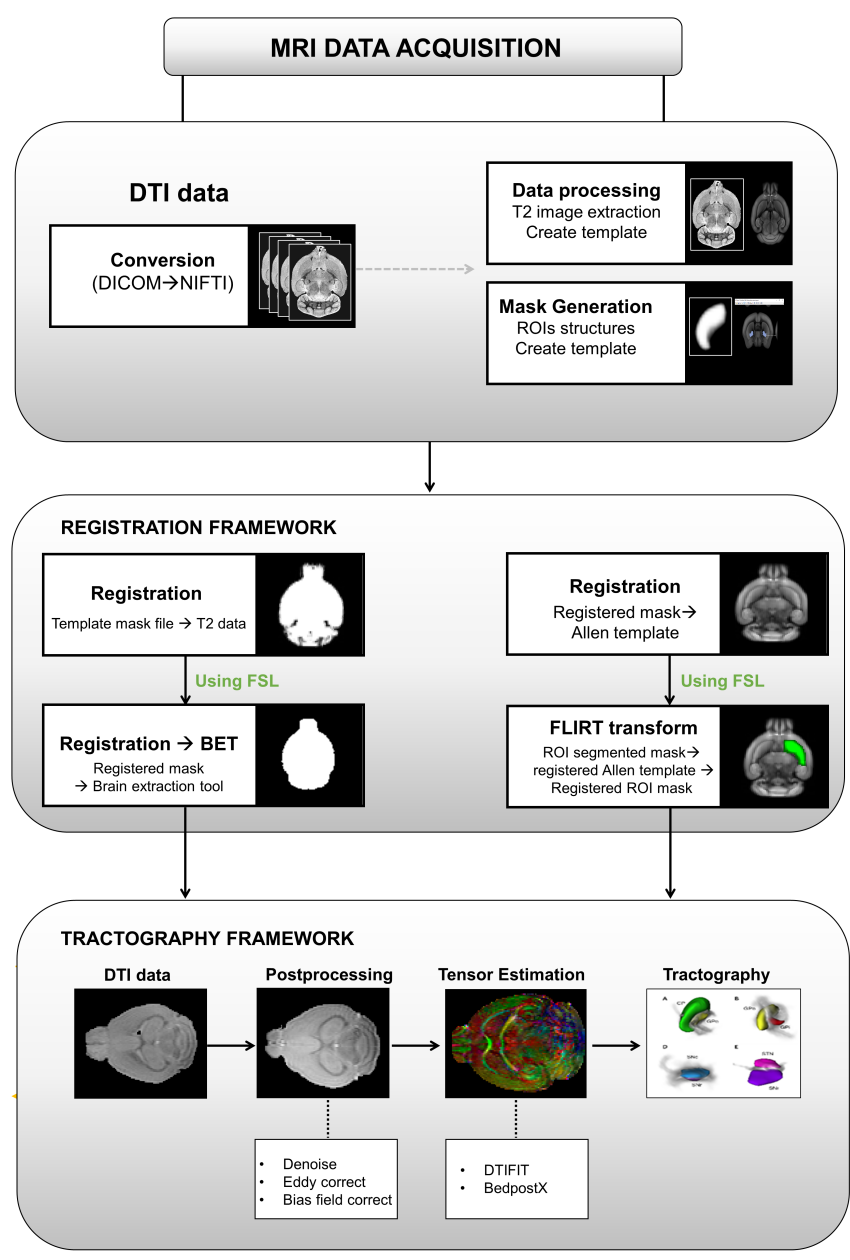

Fig. 1. Flowchart illustrating the DTI data processing pipeline.

Parameters Obtained using Sampling Techniques (BEDPOSTX) [24] was used to model crossing fibers at each voxel of the DTI data with a maximum of 3 fiber orientations per voxel. Fiber data performed by BEDPOSTX were used to generate probabilistic tractography between the previously segmented structures.

\section{Probabilistic tractography}

Probabilistic tractography analysis was performed using FSL's PROBTRACKX on a Linux workstation [37]. PROBTRACKX calculates tracking streamlines (in this case, 5,000 samples per voxel) through fiber orientations of each voxel in the segmented regions, then computes the probability of the created streamlines. The step size and curvature parameters for PROBTRACKX were independently optimized. To optimize suitable turning angles, step size was set to $1 / 20$ of the voxel size, and the curvature value was set in the range of 0.02 to 0.3 . For probabilistic tractography, step size of 0.05 and curvature of 0.1 were chosen. Probabilistic tractography maps were obtained for each of the left and right halves of the 7 
anatomic labels. Streamline fibers were generated from seed structures and target label brain masks were used as target regions for waypoint connectivity, excluding fibers that did not pass through both seed and target regions. The number of fibers generated from probabilistic tractography was used for statistical analysis.

\section{Connectivity analysis}

Probabilistic tractography streamlines stemming from 7 seed regions passing each of the 7 target regions were used to generate connectivity estimates. Streamline fibers connecting seed and target regions were summed then normalized by the seed region volume to adjust for differences in the total number of tractography seeds per structure. The streamline fibers generated between leftleft, left-right, right-left and right-right hemisphere structures were represented in a connectivity matrix, displayed with a $\log 10$ color scale.

\section{Comparison with Allen Brain Atlas neuronal tracer data and our DTI data}

To compare and visualize the differences between Allen neuronal tracer data and our DTI connectivity data, we obtained tracer injection and projection data of neuronal tracer experiments between two specific regions (such as STN-GPi) from the Allen Mouse Brain Connectivity Atlas database (http://connectivity. brain-map.org/). The database did not contain neuronal tracer data for certain region pairs such as GPe-STN. The average template image, which is in ABA reference space, is transformed into our native diffusion mouse data space using FSL's FLIRT algorithm. The ABA injection/projection data were registered to native diffusion space using the generated matrix data from the

\section{(A)}
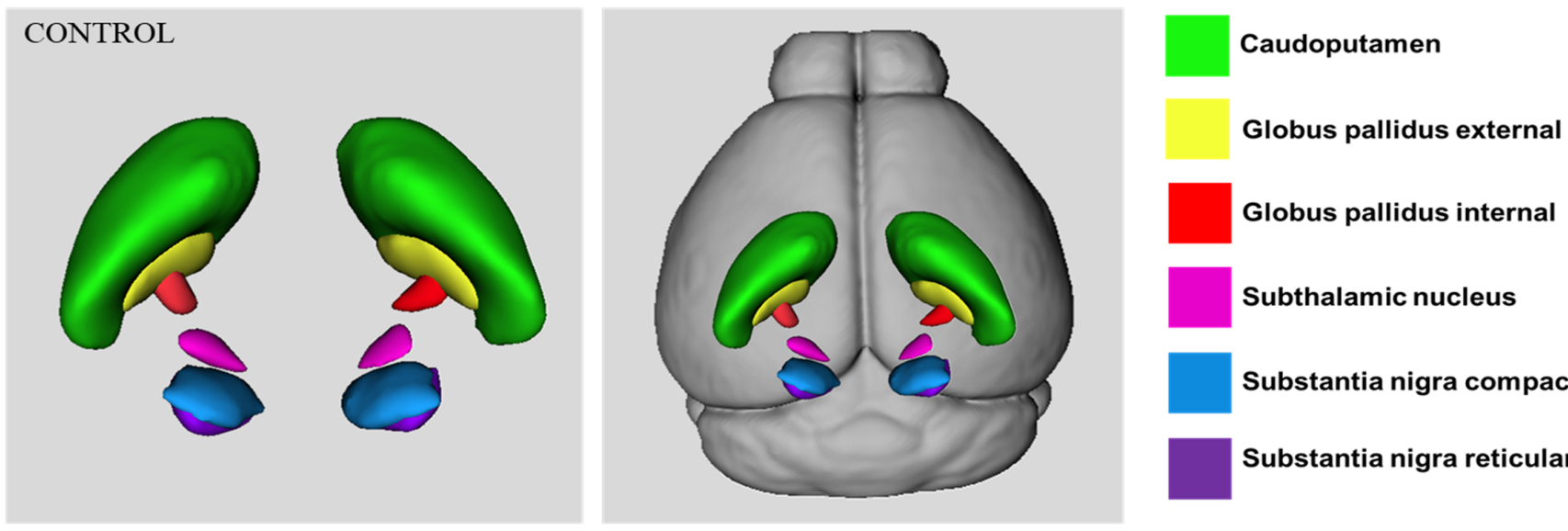

(B)
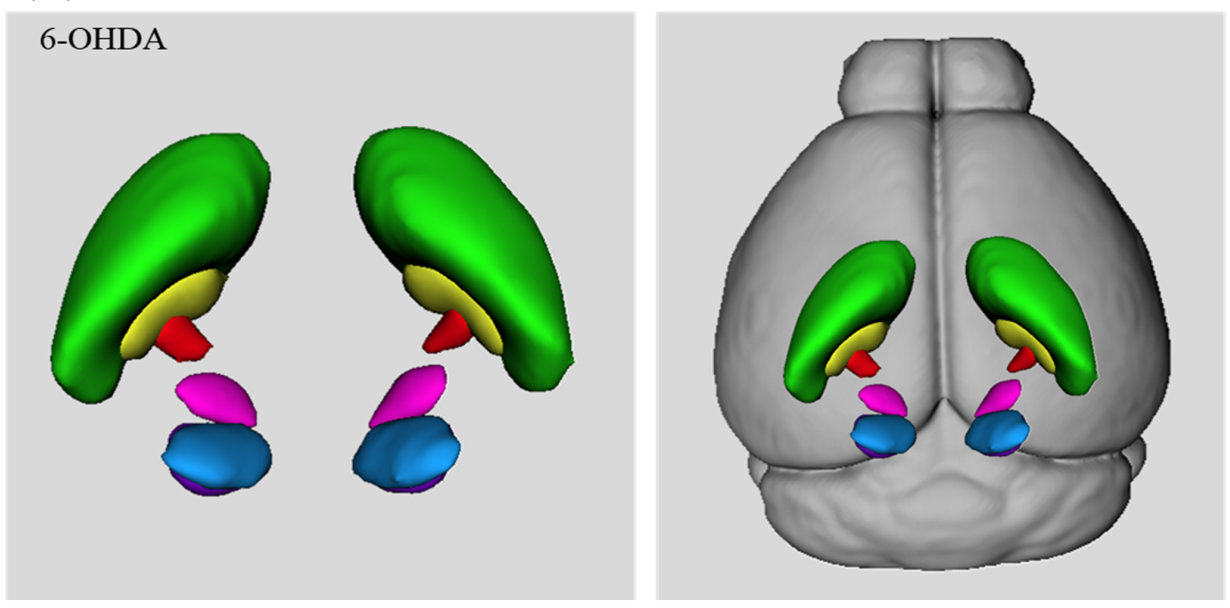

Substantia nigra compact

Substantia nigra reticular

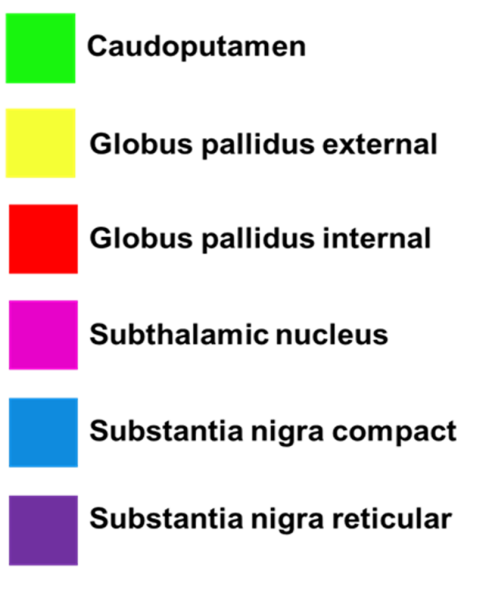

Fig. 2. 3D representations of Segmented bilateral basal ganglia structure. (A) Segmented bilateral structures in control mouse brain. (B) Segmented bilateral mouse brain in 6-OHDA-induced PD mouse model. Color code: green: caudoputamen, yellow: globus pallidus exteral, red: globus pallidus internal, pink=subthalamic nucleus, blue: substantia nigra compact, purple: substantia nigra reticular. 
transformation of average template space to diffusion space. After registration, probabilistic tractography were compared with ABA neuronal tracer data using FSL's visualization tool.

\section{Statistical analysis}

Statistical analysis was performed using Statistical Package for the Social Sciences (SPSS v 25.0, IBM Corporation, Armonk, NY, USA) software. Tractography data were tested for normality using Shapiro Wilk's test. Group comparisons between controls and 6-OHDA-induced PD mouse were done using independent samples t-test for normally distributed data and Mann-Whitney $\mathrm{U}$ test for data that were not normally distributed. A correction for multiple comparisons was performed using the Benjamini and Hochberg false discovery rate (FDR) correction with significance at $p$ values $<0.05$. Data are represented as mean and standard deviation $(\mathrm{SD})$.

\section{RESULTS}

\section{Atlas-based Segmentation}

Basal-ganglia structures segmented using ANTx for both normal (Fig. 2A) and 6-OHDA-induced PD mouse (Fig. 2B) are presented in $3 \mathrm{D}$ models for comparison. We segmented bilateral caudoputamen (CP), globus pallidus externa (GPe), globus pallidus interna (GPi), subthalamic nucleus (STN), substantia nigra compact (SNc) and substantia nigra reticular (SNr) from diffusion images. Segmented bilateral structures are represented within a 3D transparent mouse brain rendering.

\section{Brain volume}

Group-averaged segmented brain volumes from control and 6-OHDA groups in left hemisphere and right hemisphere are shown in Table 2. Estimated basal ganglia volumes of all segment- ed structures were derived from T2 data. No significant volumetric differences between control and 6-OHDA groups were found in right, as well as left hemispheres.

\section{Probabilistic tractography}

Probabilistic tractography was used to generate brain basal ganglia connectivity matrices in control (Fig. 3A) and PD-induced mouse model (Fig. 3B). The number of fibers generated from probabilistic tractography is affected by the volume of each seed region used in PROBTRACTX, which results in different levels of fiber connectivity between seed region $\mathrm{A}$, target region $\mathrm{B}$ and seed region $\mathrm{B}$, target region $\mathrm{A}$. Thus, we generated 4 different fiber connectivity matrices- 1) left seed structures to left target structures, 2) left seed structures to right target structures, 3) right seed structures to right target structures, 4) right seed structures to left target structures. In both mouse models, the fiber connectivity of the ipsilateral hemisphere was stronger than that of the contralateral connectivity.

The percentage of direct fiber connectivity between each segmented CP, GPe, GPi, SNc, SNr and STN in control and 6-OHDA groups is shown in Table 3. We averaged the number of fibers with direct connections between segmentations of both hemispheres. The percentages indicate the amount of fiber connections that pass through the target regions from the seed regions. For example, $19.79 \%$ of all streamline fibers that run through STN are connected to GP in control group mice while $21.89 \%$ in 6-OHDA group mice are connected to the GP. This table is asymmetric because it indicates the percentage of connections to target brain regions relative to the total number of fibers generated within each brain region.

Table 2. Volumes of basal ganglia of the control and 6-OHDA groups in left and right hemisphere

\begin{tabular}{|c|c|c|c|c|c|c|}
\hline & \multicolumn{6}{|c|}{ Brain volume } \\
\hline & \multicolumn{3}{|c|}{ Left hemisphere } & \multicolumn{3}{|c|}{ Right hemisphere } \\
\hline & $\begin{array}{c}\text { Control } \\
n=5\end{array}$ & $\begin{array}{c}\text { 6-OHDA } \\
n=5\end{array}$ & p value & $\begin{array}{c}\text { Control } \\
n=5\end{array}$ & $\begin{array}{c}\text { 6-OHDA } \\
n=5\end{array}$ & $p$ value \\
\hline CaudPu & $19.25 \pm 0.65$ & $19.4 \pm 0.6$ & 0.723 & $19.31 \pm 0.62$ & $19.53 \pm 0.69$ & 0.610 \\
\hline GPe & $2.93 \pm 0.09$ & $2.92 \pm 0.12$ & 0.828 & $2.88 \pm 0.15$ & $2.93 \pm 0.11$ & 0.530 \\
\hline GPi & $1.08 \pm 0.08$ & $1.07 \pm 0.06$ & 0.852 & $1.04 \pm 0.05$ & $1.07 \pm 0.1$ & 0.593 \\
\hline $\mathrm{SNc}$ & $1.65 \pm 0.07$ & $1.71 \pm 0.08$ & 0.244 & $1.68 \pm 0.05$ & $1.66 \pm 0.12$ & 0.819 \\
\hline $\mathrm{SNr}$ & $2.45 \pm 0.13$ & $2.46 \pm 0.09$ & 0.868 & $2.43 \pm 0.11$ & $2.36 \pm 0.1$ & 0.347 \\
\hline STN & $1.08 \pm 0.05$ & $1.07 \pm 0.04$ & 0.855 & $1.03 \pm 0.04$ & $1.02 \pm 0.04$ & 0.589 \\
\hline
\end{tabular}

CaudPu, caudoputamen; GPe, globus pallidus external; GPi, globus pallidus internal; SNc, substantia nigra compact; SNr, substantia nigra reticular; STN, subthalamic nucleus. 


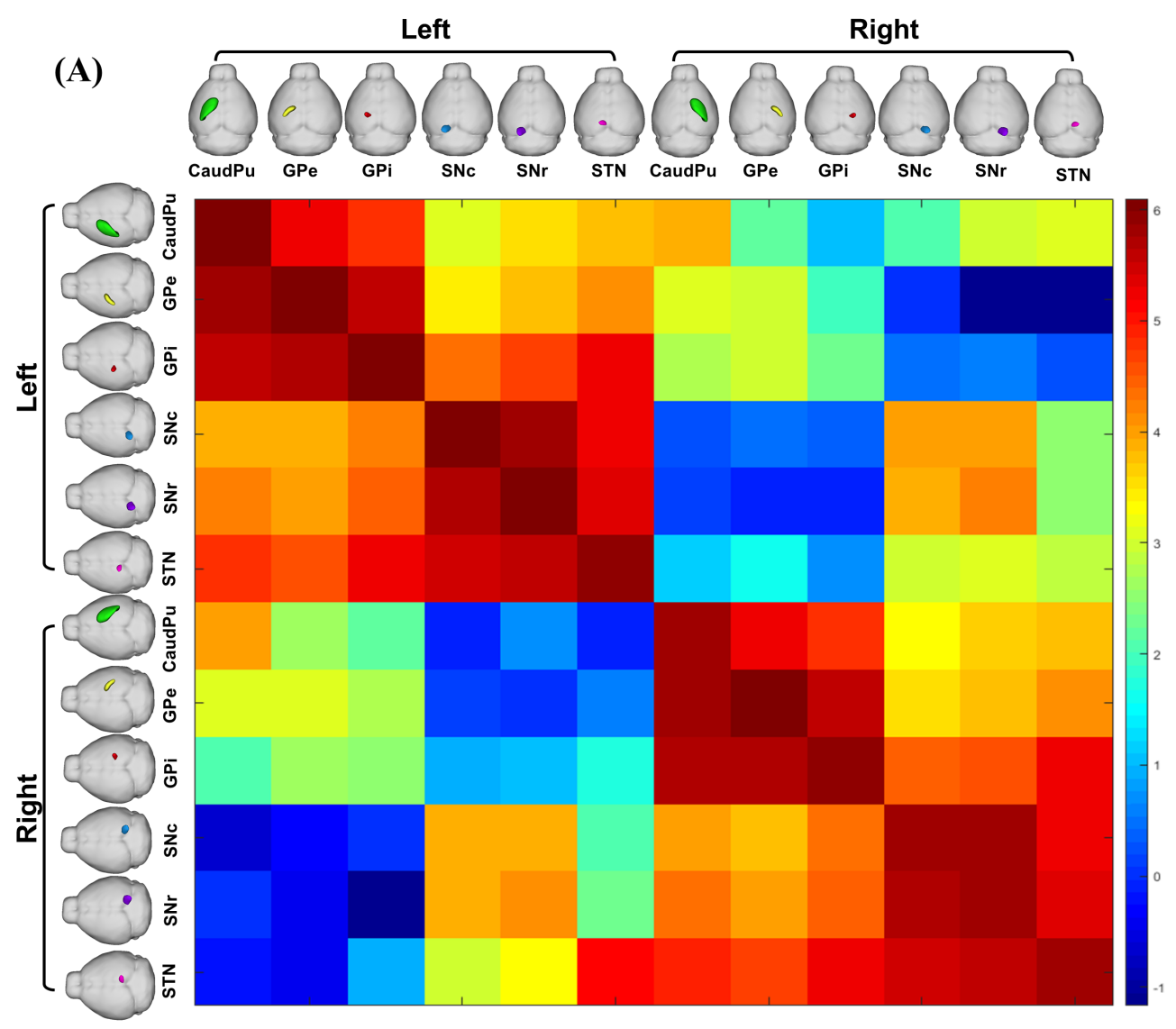

(B)

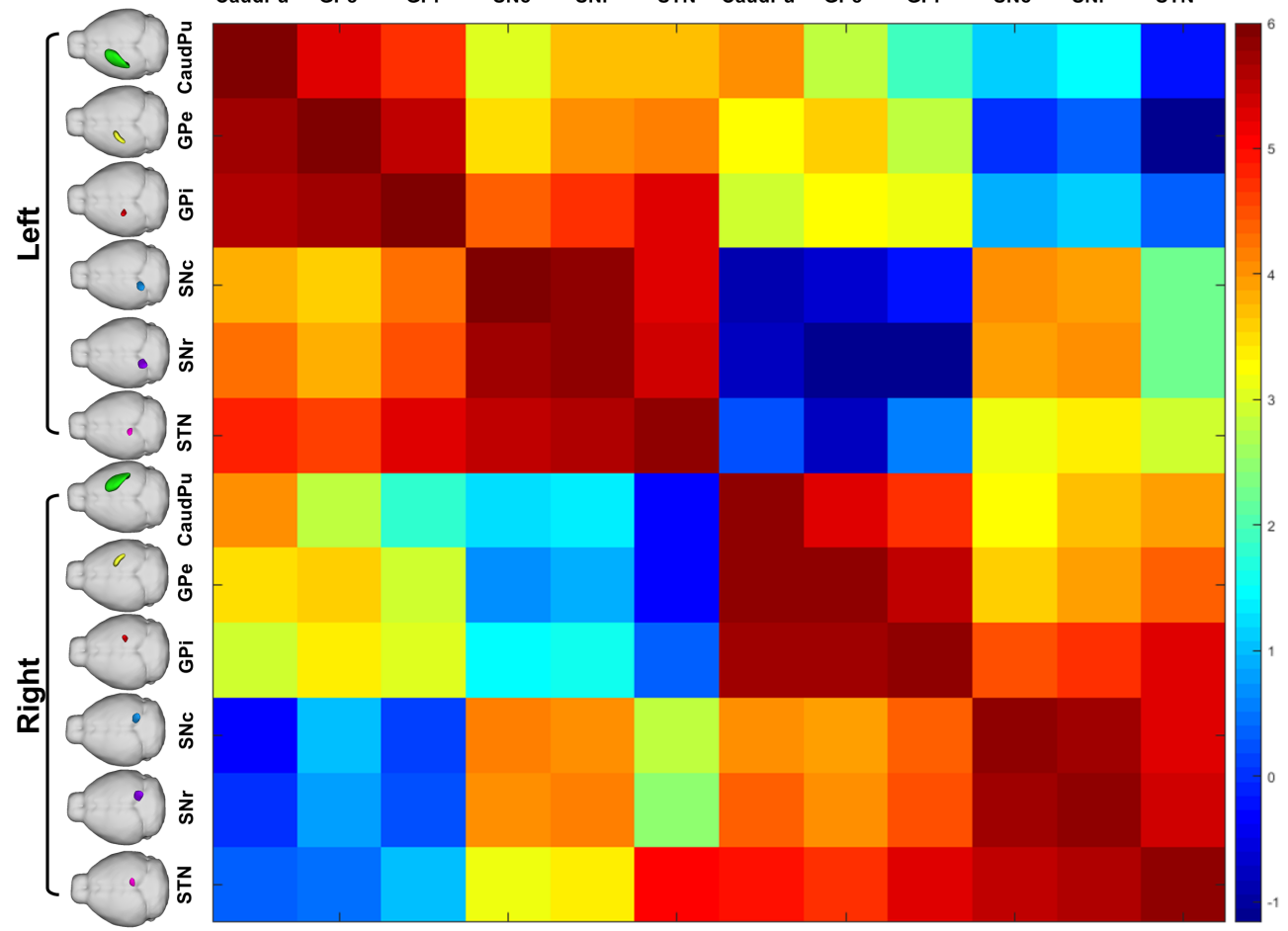

Fig. 3. A probabilistic tractography connectivity matrix between seven anatomic regions $(\mathrm{CP}$, STN, GPe, GPi, SNc, SNr, STN) displayed with a $\log 10$ scale color map for control groups (A) and 6-OHDA groups (B). The seed regions and the target regions were anatomically located within the $3 \mathrm{D}$ rendered mouse, with the seed region on the left and the target region on the top $(\mathrm{CP}$ : green, GPe: yellow, GPi: red, SNc: blue, SNr: purple, STN: pink). 
Table 3. Percentage of fiber paths directly connected from the seed structures to the target structures

\begin{tabular}{|c|c|c|c|c|c|c|c|c|c|c|c|c|}
\hline \multirow{4}{*}{$\begin{array}{c}\text { Seed } \\
\text { structures }\end{array}$} & \multicolumn{6}{|c|}{ Control group } & \multicolumn{6}{|c|}{ 6-OHDA group } \\
\hline & \multicolumn{6}{|c|}{ Target structures } & \multicolumn{6}{|c|}{ Target structures } \\
\hline & CaduPu & GPe & GPi & SNc & $\mathrm{SNr}$ & STN & CaduPu & GPe & GPi & SNc & $\mathrm{SNr}$ & STN \\
\hline & $\%(\mathrm{SD})$ & $\%$ (SD) & $\%$ (SD) & $\%$ (SD) & $\%$ (SD) & $\%(\mathrm{SD})$ & $\%(S D)$ & $\%(\mathrm{SD})$ & $\%$ (SD) & $\%$ (SD) & $\%(S D)$ & $\%(\mathrm{SD})$ \\
\hline CaudPu & - & $\begin{array}{c}70.36 \\
(15.66)\end{array}$ & $\begin{array}{l}24.63 \\
(5.49)\end{array}$ & $\begin{array}{c}0.67 \\
(0.17)\end{array}$ & $\begin{array}{l}1.77 \\
(0.4)\end{array}$ & $\begin{array}{c}2.59 \\
(0.58)\end{array}$ & - & $\begin{array}{l}70.98 \\
(15.8)\end{array}$ & $\begin{array}{l}23.65 \\
(5.29)\end{array}$ & $\begin{array}{c}0.65 \\
(0.15)\end{array}$ & $\begin{array}{c}2.04 \\
(0.46)\end{array}$ & $\begin{array}{c}2.7 \\
(0.62)\end{array}$ \\
\hline GPe & $\begin{array}{c}62.11 \\
(26.96)\end{array}$ & - & $\begin{array}{c}35.47 \\
(15.43)\end{array}$ & $\begin{array}{c}0.33 \\
(0.15)\end{array}$ & $\begin{array}{c}0.67 \\
(0.29)\end{array}$ & $\begin{array}{l}1.45 \\
(0.69)\end{array}$ & $\begin{array}{l}62.12 \\
(34.6)\end{array}$ & - & $\begin{array}{c}34.47 \\
(19.28)\end{array}$ & $\begin{array}{c}0.38 \\
(0.23)\end{array}$ & $\begin{array}{c}0.96 \\
(0.55)\end{array}$ & $\begin{array}{c}2.1 \\
(1.26)\end{array}$ \\
\hline GPi & $\begin{array}{c}38.31 \\
(20.55)\end{array}$ & $\begin{array}{c}43.08 \\
(23.05)\end{array}$ & - & $\begin{array}{l}2.05 \\
(1.1)\end{array}$ & $\begin{array}{c}3.5 \\
(1.95)\end{array}$ & $\begin{array}{l}13.09 \\
(7.03)\end{array}$ & $\begin{array}{c}37.66 \\
(23.23)\end{array}$ & $\begin{array}{c}41.64 \\
(25.55)\end{array}$ & - & $\begin{array}{c}2.3 \\
(1.48)\end{array}$ & $\begin{array}{l}3.89 \\
(2.4)\end{array}$ & $\begin{array}{l}14.54 \\
(9.0)\end{array}$ \\
\hline $\mathrm{SNc}$ & $\begin{array}{c}1.15 \\
(0.59)\end{array}$ & $\begin{array}{c}0.86 \\
(0.43)\end{array}$ & $\begin{array}{c}2.15 \\
(1.09)\end{array}$ & - & $\begin{array}{c}72.97 \\
(35.55)\end{array}$ & $\begin{array}{c}22.9 \\
(11.46)\end{array}$ & $\begin{array}{c}1.17 \\
(0.66)\end{array}$ & $\begin{array}{c}0.76 \\
(0.44)\end{array}$ & $\begin{array}{c}2.48 \\
(1.32)\end{array}$ & - & $\begin{array}{c}73.28 \\
(37.15)\end{array}$ & $\begin{array}{c}22.33 \\
(11.67)\end{array}$ \\
\hline $\mathrm{SNr}$ & $\begin{array}{c}2.45 \\
(1.41)\end{array}$ & $\begin{array}{c}1.19 \\
(0.68)\end{array}$ & $\begin{array}{c}3.28 \\
(1.86)\end{array}$ & $\begin{array}{c}63.54 \\
(34.96)\end{array}$ & - & $\begin{array}{l}29.56 \\
(13.1)\end{array}$ & $\begin{array}{c}2.83 \\
(1.56)\end{array}$ & $\begin{array}{l}1.22 \\
(0.7)\end{array}$ & $\begin{array}{c}3.91 \\
(2.16)\end{array}$ & $\begin{array}{c}63.18 \\
(33.31)\end{array}$ & - & $\begin{array}{c}28.9 \\
(15.81)\end{array}$ \\
\hline STN & $\begin{array}{c}6.83 \\
(4.12)\end{array}$ & $\begin{array}{c}3.79 \\
(2.23)\end{array}$ & $\begin{array}{c}16 \\
(9.57)\end{array}$ & $\begin{array}{c}31.74 \\
(18.47)\end{array}$ & $\begin{array}{c}41.66 \\
(24.21)\end{array}$ & - & $\begin{array}{c}7.16 \\
(4.54)\end{array}$ & $\begin{array}{c}3.91 \\
(2.47)\end{array}$ & $\begin{array}{l}17.98 \\
(11.3)\end{array}$ & $\begin{array}{c}30.68 \\
(19.05)\end{array}$ & $\begin{array}{c}40.29 \\
(24.99)\end{array}$ & - \\
\hline
\end{tabular}

CaudPu, caudoputamen; GPe, globus pallidus external; GPi, globus pallidus internal; SNc, substantia nigra compact; SNr, substantia nigra reticular; STN, subthalamic nucleus.

(A)

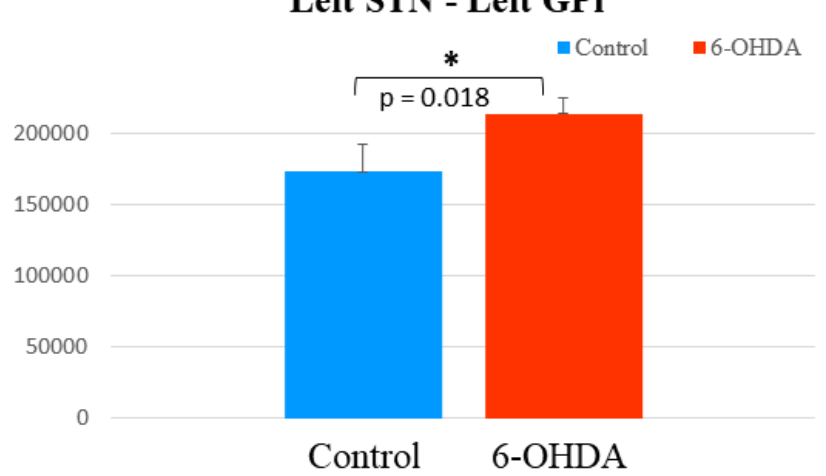

(B)

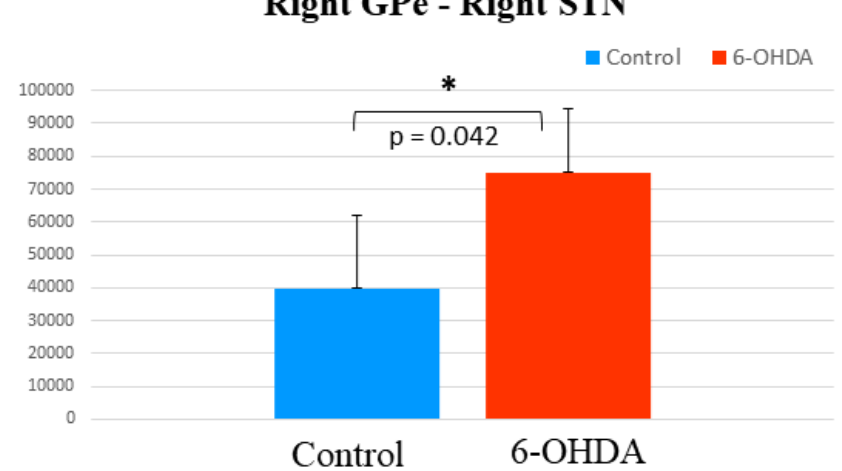

Fig. 4. Comparison graphs of direct connections between control and 6-OHDA groups in left STN-left GPi and right GPe-right STN connectivity. Mean and standard deviation. ${ }^{*} \mathrm{p}<0.05$ significant differences after adjusting for FDR correction (Blue: control group, Red: 6-OHDA group).

\section{Significant direct probabilistic tractography within the basal ganglia}

We identified basal ganglia connectivity with significant differences between the two groups through fiber path data obtained through FSL's PROBTRACKX. After testing for normality and differences between the two groups, we found that left STN - left GPi (Fig. 4A) and right GPe - right STN (Fig. 4B) connectivity showed significant differences between control and 6-OHDA group. Group-averaged left STN - left GPi connection was higher in 6-OHDA group than in control group $(\mathrm{p}=0.018)$. Moreover, right GPe - right STN connection was also higher in 6-OHDA group ( $\mathrm{p}=0.042)$ than in control group. However, there were no obvious significant differences in fiber connectivity in other brain structures that were analyzed.

\section{Increased subthalamo-pallidal and pallido-subthalamic connectivity in PD model}

Waypoint probabilistic connectivity maps were generated by PROBTRACKX. PROBTRACKX visualizes the streamline fibers connecting seed and target structures in grey $3 \mathrm{D}$ meshes. We displayed detailed tracts connecting STN-GPi and GPe-STN of both control and 6-OHDA-injected PD mouse group, shown in Fig. 5. 

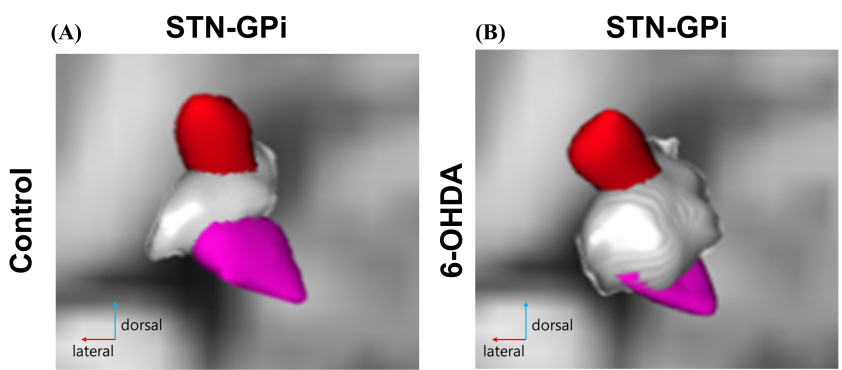

(C)

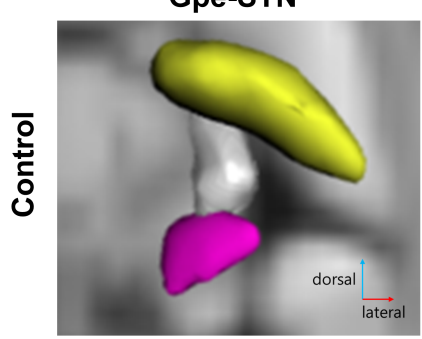

(D)

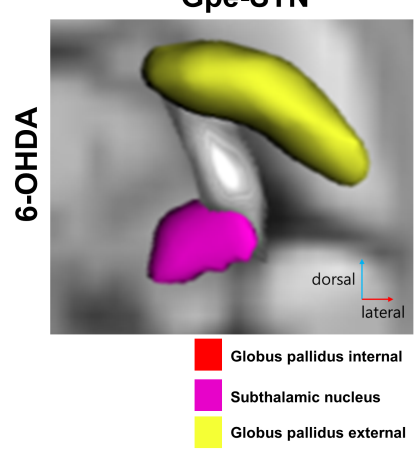

Fig. 5. Detailed fiber connectivity between seed region and target region in control and 6-OHDA groups. This figure represents a 3D dorsal view of STN-GPi and GPe-STN tracts on an axial T2-weighted image. (A) and (B) show tracts interconnecting the left STN and the left GPi in control and 6-OHDA groups. (C) and (D) shows tracts interconnecting the right GPe and right STN in control and 6-OHDA groups (Red: GPi, Pink: STN, Gray: fiber path; (A) Left STN-left GPi in control,(B) Left STN-left GPi in 6-OHDA, (C) Right GPe-right STN in control, (D) Right GPe-right STN in 6-OHDA)

In left STN - left GPi connectivity (Fig. 5A and 5B), most of the fibers extend from the dorsal-medial aspect of the STN. However, we found that dorsal-lateral and medial fibers of the STN are denser in the 6-OHDA group than in the control group. In right GPe - right STN connectivity (Fig. 5C and 5D), most fibers reconstructed using PROBTRACKX emerged from the ventral-medial region of GPe in both groups, whereas more fibers of dorsallateral parts of STN were innervated by ventral-medial GPe in the 6-OHDA group.

\section{Comparison of probabilistic tractography and Allen Brain Atlas neuronal tracer data}

Probabilistic tractography data was compared with ABA neuronal tracer data. Diffusion-based probabilistic tractography were obtained from ROIs corresponding to ABA tracer injection sites. Probabilistic tractography data were used to investigate for the accuracy of using diffusion probabilistic tractography for mapping structural fiber connectivity between basal ganglia regions (Fig. 6A). We compared ABA fiber projections with our tractography data that extends to GPi from STN as injection sites, shown in Fig.
6B. The STN-GPi projection data from ABA was generated from C57BL/6J mouse. Although probabilistic tractography data did not correspond completely with ABA neuronal tracer data, we found that more fibers in the 6-OHDA mouse model were distributed in a similar medial direction as neuronal tracer data.

\section{DISCUSSION}

In this work, we identified the differences in basal ganglia connectome for ex-vivo mouse with 6-OHDA-induced PD model versus normal groups. We presented that by using high-resolution diffusion MRI data at 9.4 Tesla, we are able to detect complex structural fiber connectivity and to reconstruct maps by using the fiber information from the voxel neighborhood in the basal ganglia. We previously structured the analysis pipeline on ex-vivo mouse diffusion data for delineating well-known fiber connections involving Parkinson's disease [37]. To obtain a more refined view of the diffusion orientations, we applied a further refined fiber tracking algorithm with postprocessing steps; this allowed us to generate higher resolution diffusion orientations. In addition, we used probabilistic tractography to infer connectivity of the seed and target regions and detect a larger number of fibers. This process provides information on comparative mapping of the basal ganglia connectivity in PD mouse model and wild-type mouse model.

\section{Brain volume}

We detected no basal ganglia volume change between 6-OHDA and control mouse models using FSL analysis methods. Our findings support the results of previous studies that did not find subcortical brain volume (including basal ganglia and thalamus) changes [38-40]. Rektor et al. [40] study also did not detect subcortical volumetric changes in early stage PD patents, suggesting that alterations of brain volume may occur late stage in the disease course. Indeed, a number of studies detected volumetric abnormalities of part of subcortical structure (e.g. striatum and thalamus) in advanced PD patients, indicating that subcortical volumetric atrophy occurs later stage during the disease course [40, 41].

\section{Tractography of the basal ganglia in ex-vivo PD mouse and normal mouse}

Using probabilistic tractography analysis, we obtained connectivity matrix for the segmented basal ganglia. Furthermore, direct fiber connections within the brain structures were found in control and 6-OHDA groups. The overall number of fibers on the contralateral hemisphere were lower than fibers on the ipsilateral 
(A)

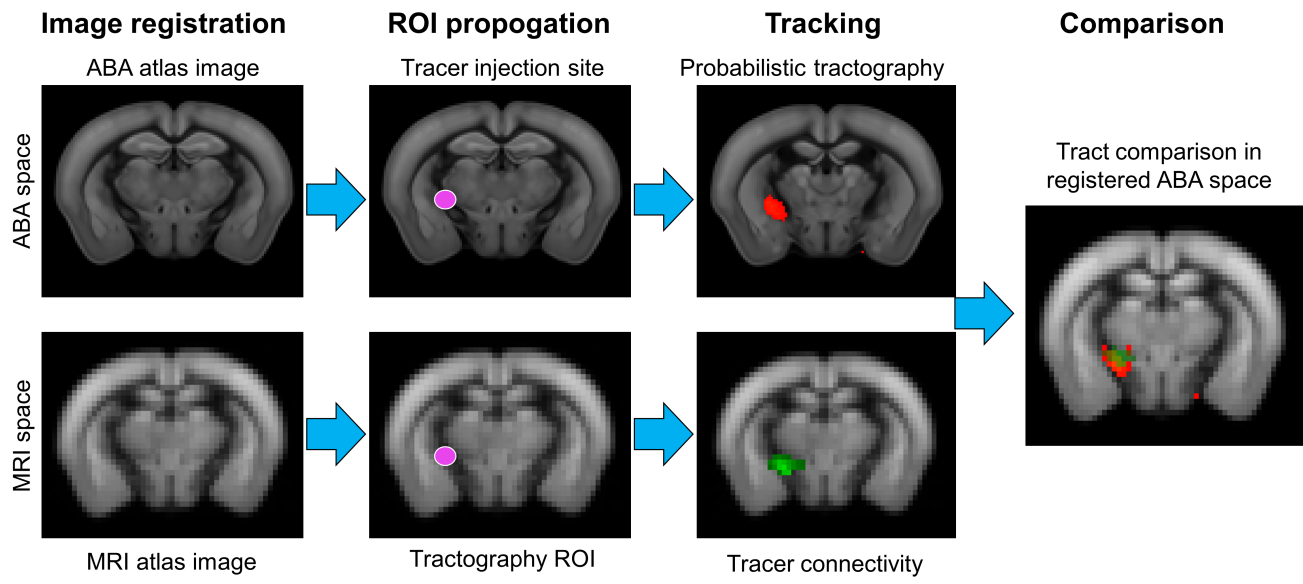

(B) Control

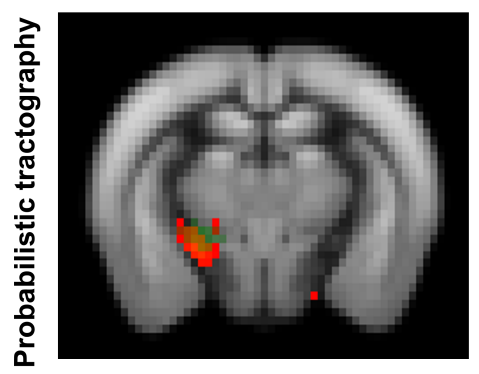

6-OHDA

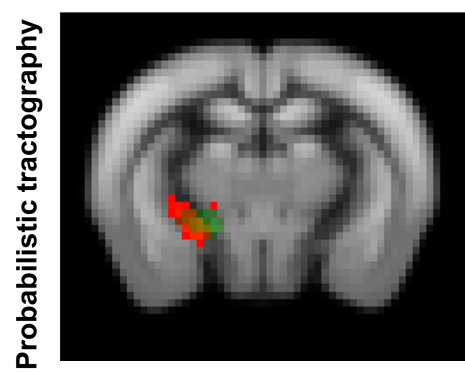

Fig. 6. Comparison between ABA neuronal tracer and diffusion tractography projection. (A) The ABA neuronal projections (colored in red) from the injection site were registered to $\mathrm{ABA}$ mouse brain template. ABA brain template (along with the neuronal projections) was registered to our native MR mouse image, where probabilistic fiber data (colored in green) were compared with $\mathrm{ABA}$ neuronal tracer projections. (B) Probabilistic tractography (green) and $\mathrm{ABA}$ neuronal projections of STNGPi in control and 6-OHDA mouse model. hemisphere. The projections of ipsilateral and contralateral hemispheres showed similar patterns. The pattern of contralateral connectivity appears to be an attenuated version of the ipsilateral side pattern. These findings are in line with previous human DTI study which demonstrated a bilateral connectivity in cerebellum-basal ganglia. The weaker projections observed for the contralateral side connections may be due to the longer distance necessary for probabilistic pathway to reach the final target region [22]. Moreover, these findings indicate that basic topological organization of contralateral and ipsilateral connectivity is similar between human and mouse.

As mentioned in the above results, the connectivity between two short distance brain regions is higher because the tract can be measured more easily than the longer distance connection. For example, the STN-GPi connections (16\% of all fibers through the STN in control group, $17.98 \%$ of all fibers through the STN in 6-OHDA group) are higher than the STN-GPe connections (3.79\% in control, 3.91\% in 6-OHDA group). This may suggest that the STN-GPi connectivity could be overestimated because of distance proximity [16]. Overall, we found reproducible connectivity estimates across basal ganglia for both left and right hemispheres in each individual mouse models.

\section{Subthalamo-pallidal and pallido-subthalamic pathway}

We identified that left STN-left GPi and right GPe-right STN connectivity are significantly increased in 6-OHDA group compared to control. The STN and GPi are the main targets for the treatment of movement disorder because of their effectiveness post-surgical treatment [42]. After dopamine depletion in PD, indirect pathway (GP and STN) excessively inhibits the motor loop circuit, resulting in rigidity of movement [43]. In present study, a large number of fibers extended from the dorsal-medial region of the STN to GPi. This finding is in line with a previous in-vivo human brain study using multi-fiber tractography [8]. However, in 6-OHDA mouse models, more fibers were distributed in dorsal-lateral and medial regions of the STN to GPi. The large dorsal-lateral area of STN is part of the motor territory; ventralmedial area corresponds to the associative territory and medial area corresponds to the limbic territory [44]. One functional MRI study reported that increased alteration in STN fiber connectivity is associated with changes to basal ganglia motor circuit in the PD group. These results can be inferred that STN-related connectivity is increased as a compensatory response to control movement during self-initiated movement [45]. Although functional connectivity does not indicate direct structural connectivity, detection of 
overlapping regions in both structural and functional techniques imply a strong indication of the reliability in the proposed model [14].

Furthermore, we found that the GPe-STN connections displayed significant increases in 6-OHDA group. The topologic distribution of this projection varies from species to species. In agreement with previous rodent studies, we identified that dorsal-lateral STN is innervated by the ventral-medial pallidum [46]. However, more alterations in fibers between GPe and STN have been demonstrated in 6-OHDA mouse model. In PD models with dopaminergic depletion, the pallido-subthalamic projections are enhanced by an increase in the number of fiber synaptic connections from the GPe terminals to the STN. Such increase in inhibition projections from the GPe may induce hyperpolarization-mediated rebound bursting of STN cells [47]. Thus, data on network alterations could contribute to studying abnormal activity patterns of parkisonian STN.

\section{Allen neuronal tracer data}

While probabilistic tractography can provide estimates of connectivity between basal ganglia structures and cerebral cortex, such results may have some limitations due to potential underestimates referred from prior studies on fiber tracts between mouse basal ganglia [48]. Therefore, we compared our tractography data with Allen mouse neuronal tracer data to evaluate the accuracy of using diffusion probabilistic tractography for mapping basal ganglia connectivity. Although the results presented here showed that more fibers were distributed in the medial region of STN in 6-OHDA models than in controls, the data of both groups did not completely agree with ABA tracer data. This is likely due to the fundamental differences between tractography and neuronal tracer data. Calabrese study explained that this may be due to differences in the properties of the anterograde viral tracers that cannot pass through synapses and restricted to moving unidirectionally through individual axons, unlike that of tractography, which can be generated in multiple-directions through bundles of axons and in between synapses [10].

\section{Limitations}

We recognize that the present study has some limitations. The direction of the connectivity cannot be inferred with probabilistic tractography techniques. Therefore, it is important to know that the generated tracts do not reflect the projection directionality. For example, GPe-STN connections may also reflect STN-GPe connections. Second, it cannot be inferred whether the obtained fiber projections are inhibitory or excitatory. Finally, the biological interpretation of results remains a challenge as connectivity derived diffusion neuronal tractography can only be inferred and may require further studies with similar mouse models for verification.

\section{Conclusion}

In conclusion, the current study demonstrated that 6-OHDAinjected PD mouse shows alterations on motor-related basal ganglia using probabilistic tractography. We found that subthalamopallidal and pallido-subthalamic connectivity were strengthened in PD mouse model. Abnormal fiber projections within the STN are associated with motor territory, which remains an important region of the basal ganglia in the treatment and pathophysiology of Parkinson's disease. In this context, our ex-vivo mouse brain connectivity can potentially be a crucial model for future mouse brain studies in understanding mechanisms of several neurologic and psychiatric diseases.

\section{ACKNOWLEDGEMENTS}

This research was supported by Brain Research Program through the National Research Foundation of Korea (NRF) funded by the Ministry of Science and ICT (NRF-2017m3c7a1044367).

\section{REFERENCES}

1. Pavese N, Brooks DJ (2009) Imaging neurodegeneration in Parkinson's disease. Biochim Biophys Acta 1792:722-729.

2. Caligiore D, Helmich RC, Hallett M, Moustafa AA, Timmermann L, Toni I, Baldassarre G (2016) Parkinson's disease as a system-level disorder. NPJ Parkinsons Dis 2:16025.

3. Joe EH, Choi DJ, An J, Eun JH, Jou I, Park S (2018) Astrocytes, microglia, and Parkinson's disease. Exp Neurobiol 27:77-87.

4. Son SJ, Kim M, Park H (2016) Imaging analysis of Parkinson's disease patients using SPECT and tractography. Sci Rep 6:38070.

5. Villalba RM, Mathai A, Smith Y (2015) Morphological changes of glutamatergic synapses in animal models of Parkinson's disease. Front Neuroanat 9:117.

6. DeLong MR, Wichmann T (2007) Circuits and circuit disorders of the basal ganglia. Arch Neurol 64:20-24.

7. Müller HP, Kassubek J (2013) Diffusion tensor magnetic resonance imaging in the analysis of neurodegenerative diseases. JVis Exp 77:e50427.

8. Pujol S, Cabeen R, Sébille SB, Yelnik J, François C, Fernandez Vidal S, Karachi C, Zhao Y, Cosgrove GR, Jannin P, Kikinis R, Bardinet E (2017) In vivo exploration of the connectivity between the subthalamic nucleus and the globus pallidus in the human brain using multi-fiber tractography. Front Neu- 
roanat 10:119.

9. Basser PJ, Mattiello J, LeBihan D (1994) Estimation of the effective self-diffusion tensor from the NMR spin echo. J Magn Reson B 103:247-254.

10. Calabrese E, Badea A, Cofer G, Qi Y, Johnson GA (2015) A diffusion MRI tractography connectome of the mouse brain and comparison with neuronal tracer data. Cereb Cortex 25:4628-4637.

11. Cong L, Muir ER, Chen C, Qian Y, Liu J, Biju KC, Clark RA, Li S, Duong TQ (2016) Multimodal MRI evaluation of the MitoPark mouse model of Parkinson's disease. PLoS One 11:e0151884.

12. Hübner NS, Mechling AE, Lee HL, Reisert M, Bienert T, Hennig J, von Elverfeldt D, Harsan LA (2017) The connectomics of brain demyelination: functional and structural patterns in the cuprizone mouse model. Neuroimage 146:1-18.

13. Perlbarg V, Lambert J, Butler B, Felfli M, Valabrègue R, Privat AL, Lehéricy S, Petiet A (2018) Alterations of the nigrostriatal pathway in a 6-OHDA rat model of Parkinson's disease evaluated with multimodal MRI. PLoS One 13:e0202597.

14. Lenglet C, Abosch A, Yacoub E, De Martino F, Sapiro G, Harel $\mathrm{N}$ (2012) Comprehensive in vivo mapping of the human basal ganglia and thalamic connectome in individuals using 7T MRI. PLoS One 7:e29153.

15. Milardi D, Arrigo A, Anastasi G, Cacciola A, Marino S, Mormina E, Calamuneri A, Bruschetta D, Cutroneo G, Trimarchi F, Quartarone A (2016) Extensive direct subcortical cerebellum-basal ganglia connections in human brain as revealed by constrained spherical deconvolution tractography. Front Neuroanat 10:29.

16. Plantinga BR, Roebroeck A, Kemper VG, Uludağ K, Melse M, Mai J, Kuijf ML, Herrler A, Jahanshahi A, Ter Haar Romeny BM, Temel Y (2016) Ultra-high field MRI post mortem structural connectivity of the human subthalamic nucleus, substantia nigra, and globus pallidus. Front Neuroanat 10:66.

17. Rubinov M, Sporns O (2010) Complex network measures of brain connectivity: uses and interpretations. Neuroimage 52:1059-1069.

18. Fillard P, Descoteaux M, Goh A, Gouttard S, Jeurissen B, Malcolm J, Ramirez-Manzanares A, Reisert M, Sakaie K, Tensaouti F, Yo T, Mangin JF, Poupon C (2011) Quantitative evaluation of 10 tractography algorithms on a realistic diffusion MR phantom. Neuroimage 56:220-234.

19. Stucht D, Danishad KA, Schulze P, Godenschweger F, Zaitsev M, Speck O (2015) Highest resolution in vivo human brain MRI using prospective motion correction. PLoS One 10:e0133921.
20. Zhang J (2010) Diffusion tensor imaging of white matter pathology in the mouse brain. Imaging in medicine. Imaging Med 2:623-632.

21. Alomair OI, Brereton IM, Smith MT, Galloway GJ, Kurniawan ND (2015) In vivo high angular resolution diffusionweighted imaging of mouse brain at 16.4 Tesla. PLoS One 10:e0130133.

22. Cacciola A, Calamuneri A, Milardi D, Mormina E, Chillemi G, Marino S, Naro A, Rizzo G, Anastasi G, Quartarone A (2017) A connectomic analysis of the human basal ganglia network. Front Neuroanat 11:85.

23. Moldrich RX, Pannek K, Hoch R, Rubenstein JL, Kurniawan ND, Richards LJ (2010) Comparative mouse brain tractography of diffusion magnetic resonance imaging. Neuroimage 51:1027-1036.

24. Behrens TE, Berg HJ, Jbabdi S, Rushworth MF, Woolrich MW (2007) Probabilistic diffusion tractography with multiple fibre orientations: what can we gain? Neuroimage 34:144-155.

25. Tsai SY (2018) Reproducibility of structural brain connectivity and network metrics using probabilistic diffusion tractography. Sci Rep 8:11562.

26. Cicchetti F, Drouin-Ouellet J, Gross RE (2009) Environmental toxins and Parkinson's disease: what have we learned from pesticide-induced animal models? Trends Pharmacol Sci 30:475-483.

27. Thiele SL, Warre R, Nash JE (2012) Development of a unilaterally-lesioned 6-OHDA mouse model of Parkinson's disease. J Vis Exp 60:3234.

28. Oh SW, Harris JA, Ng L, Winslow B, Cain N, Mihalas S, Wang Q, Lau C, Kuan L, Henry AM, Mortrud MT, Ouellette B, Nguyen TN, Sorensen SA, Slaughterbeck CR, Wakeman W, Li Y, Feng D, Ho A, Nicholas E, Hirokawa KE, Bohn P, Joines KM, Peng H, Hawrylycz MJ, Phillips JW, Hohmann JG, Wohnoutka P, Gerfen CR, Koch C, Bernard A, Dang C, Jones AR, Zeng H (2014) A mesoscale connectome of the mouse brain. Nature 508:207-214.

29. Koch S, Mueller S, Foddis M, Bienert T, von Elverfeldt D, Knab F, Farr TD, Bernard R, Dopatka M, Rex A, Dirnagl U, Harms C, Boehm-Sturm P (2019) Atlas registration for edema-corrected MRI lesion volume in mouse stroke models. J Cereb Blood Flow Metab 39:313-323.

30. Cui Z, Zhong S, Xu P, He Y, Gong G (2013) PANDA: a pipeline toolbox for analyzing brain diffusion images. Front Hum Neurosci 7:42.

31. Smith SM (2002) Fast robust automated brain extraction. Hum Brain Mapp 17:143-155.

32. Jenkinson M, Smith S (2001) A global optimisation method 
for robust affine registration of brain images. Med Image Anal 5:143-156.

33. Veraart J, Novikov DS, Christiaens D, Ades-Aron B, Sijbers J, Fieremans E (2016) Denoising of diffusion MRI using random matrix theory. Neuroimage 142:394-406.

34. Andersson JLR, Sotiropoulos SN (2016) An integrated approach to correction for off-resonance effects and subject movement in diffusion MR imaging. Neuroimage 125:10631078.

35. Visser MM, Yassi N, Campbell BCV, Desmond PM, Davis SM, Spratt N, Parsons M, Bivard A (2019) White matter degeneration after ischemic stroke: a longitudinal diffusion tensor imaging study. J Neuroimaging 29:111-118.

36. Jenkinson M, Beckmann CF, Behrens TE, Woolrich MW, Smith SM (2012) FSL. Neuroimage 62:782-790.

37. Shim JH, Im SJ, Kim AY, Kim YT, Kim EB, Baek HM (2019) Generation of mouse basal ganglia diffusion tractography using 9.4T MRI. Exp Neurobiol 28:300-310.

38. Péran P, Cherubini A, Assogna F, Piras F, Quattrocchi C, Peppe A, Celsis P, Rascol O, Démonet JF, Stefani A, Pierantozzi M, Pontieri FE, Caltagirone C, Spalletta G, Sabatini U (2010) Magnetic resonance imaging markers of Parkinson's disease nigrostriatal signature. Brain 133:3423-3433.

39. Schulz JB, Skalej M, Wedekind D, Luft AR, Abele M, Voigt K, Dichgans J, Klockgether T (1999) Magnetic resonance imaging-based volumetry differentiates idiopathic Parkinson's syndrome from multiple system atrophy and progressive supranuclear palsy. Ann Neurol 45:65-74.

40. Rektor I, Svátková A, Vojtíšek L, Zikmundová I, Vaníček J, Király A, Szabó N (2018) White matter alterations in Parkinson's disease with normal cognition precede grey matter atrophy. PLoS One 13:e187939.
41. Lee SH, Kim SS, Tae WS, Lee SY, Choi JW, Koh SB, Kwon DY (2011) Regional volume analysis of the Parkinson disease brain in early disease stage: gray matter, white matter, striatum, and thalamus. AJNR Am J Neuroradiol 32:682-687.

42. Follett KA, Weaver FM, Stern M, Hur K, Harris CL, Luo P, Marks WJ Jr, Rothlind J, Sagher O, Moy C, Pahwa R, Burchiel K, Hogarth P, Lai EC, Duda JE, Holloway K, Samii A, Horn S, Bronstein JM, Stoner G, Starr PA, Simpson R, Baltuch G, De Salles A, Huang GD, Reda DJ; CSP 468 Study Group (2010) Pallidal versus subthalamic deep-brain stimulation for Parkinson's disease. N Engl J Med 362:2077-2091.

43. Loucif KC, Wilson CL, Baig R, Lacey MG, Stanford IM (2005) Functional interconnectivity between the globus pallidus and the subthalamic nucleus in the mouse brain slice. J Physiol 567:977-987.

44. Benarroch EE (2008) Subthalamic nucleus and its connections: anatomic substrate for the network effects of deep brain stimulation. Neurology 70:1991-1995.

45. Jia Q, Gao L, Zhang J, Wu T, Chan P (2018) Altered functional connectivity of the subthalamic nucleus during self-initiated movement in Parkinson's disease. J Neuroradiol 45:249-255.

46. Hamani C, Saint-Cyr JA, Fraser J, Kaplitt M, Lozano AM (2004) The subthalamic nucleus in the context of movement disorders. Brain 127:4-20

47. Fan KY, Baufreton J, Surmeier DJ, Chan CS, Bevan MD (2012) Proliferation of external globus pallidus-subthalamic nucleus synapses following degeneration of midbrain dopamine neurons. J Neurosci 32:13718-13728.

48. Vasques X, Richardet R, Hill SL, Slater D, Chappelier JC, Pralong E, Bloch J, Draganski B, Cif L (2015) Automatic target validation based on neuroscientific literature mining for tractography. Front Neuroanat 9:66. 